\title{
Decarboxylation Of Palm And Groundnut Oils In Medium Uv Irradiation
}

\author{
K.I. Ekpenyong*, N.C. Anyanwu and S. Sanusi \\ Department of Chemistry, University of Jos, P.M.B. 2084 Jos, Nigeria.
}

\begin{abstract}
Conventional palm and groundnut cooking oils have been photolysed using a medium UV photochemical lamp. Room temperature photolysis of crude palm oil led principally to decarboxylation of the residual free fatty acid. $\mathrm{H}_{2} \mathrm{O}$ treated palm oil, however, produced mainly additional fatty acid on photolysis. Decarboxylation of untreated palm oil achieved a 95\% reduction in baseline free fatty acid value within the first 10 minutes. This was followed by a gradual and linear drop in the acid value below the baseline value. Decarboxylation was also the major reaction in the acetone-sensitised crude palm oil photolysis. The decarboxylation rate was, however, slightly lower than the unsensitised reaction. In the case of groundnut oil, unsensitised and acetone-sensitised system showed similar behaviour within the first 10 minutes. For the unsensitised system, a substantial rise in acid value above baseline value was followed by decarboxylation to an acid value well below baseline in 60 minutes. For the sensitized system, the acid value remained constant at about 59\% above baseline value in the 10 to 30 minutes reaction interval. Beyond 30 minutes, it rose by as much as 155\% above baseline. The rise then slowed down somewhat after 45 minutes of reaction.
\end{abstract}

\section{INTRODUCTION}

Vegetable oils undergo appreciable deterioration during processing and storage. The residual free fatty acid (FFA) concentration of vegetable oils is a function of the processing and storage conditions. Vegetable oils spoilage, usually defined as rancidity, is the result of fatty acid moity has been recognized as the point of attack in peroxide formation ${ }^{2}$

Peroxide-forming reactions are known to involve free residual ${ }^{3,4}$ and studies have also shown singlet oxygen to be involved ${ }^{5,6}$. Dyes and pigments have also been shown to sensitize the formation of peroxides. ${ }^{7,8}$

A new dimension in vegetable oils deterioration different from the familiar and well studied reaction such as acid/base hydrolysis, and cleavage resulting from peroxide formation appears to be the case in ultraviolet (UV) irradiation of these oils.

The principal focus of our earlier studies was the decarboxylation phase of these reactions, in which, through monitoring of the $\mathrm{CO}_{2}$ evolution, the reaction rate was determined to be first order with respect to the oil concentration. In the present studies, we have been 
interested in determining how these same reactions behave in shorter time range

photolysis, e.g. during the first few

* Author for correspondence
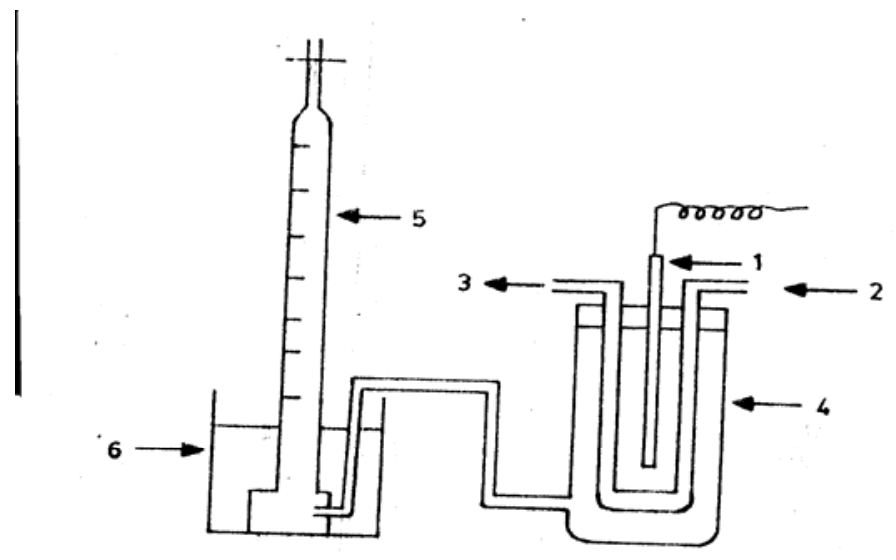

\section{EXPERIMENTAL}

Fic.I Equipment set - up for Photolysis

Lamp(I); Coolant (2/3); Reactor (4); $\mathrm{CO}_{2}$ Collection (5); Trough for displaced
The apparatus (figure 1) consisted of a low energy fluorescent lamp (product of philips company, Holland) immersed in a tube, which was itself surrounded by another tube through which cooling water circulated. A gas collection unit in form of an inverted, water filled burette, was connected by a rubber tubing to the reaction chamber. The lamp, $23.4 \times 1 \mathrm{~cm}$ in dimension, generated radiation of intensity comparable to that of the typical household fluorescent lamp. This was connected to the main power supply through a voltage regulator.

For each experimental run, the weighed oil sample (80g in most cases) was inserted in the reaction chamber, the lamplcooling unit immersed therein and the system stoppered as air-tight as possible. Power supply was then turned on commence with the experiment. In the case of acetonesesitised reactions, each oil sample was treated with acetone in the ratio of 2:3 (v/w) acetone to oil, prior to introduction into the reaction chamber. Reaction progress was monitored by measurement of the co2 evolved (in the case of decarboxylation) and by titration of the oil sample with $2 \mathrm{~m}$ aqueous alcoholic $\mathrm{NaOH}$ solution. The acidity of the oil is reported here as acid value (AV). Prior to each run, the base line AV of the oil was similarly determined.

\section{RESULT AND DISCUSSION}

figure 2 shows the result of crude palm oil both unsensitised and acetonesensitised photolysis. Figure 3 is the result obtain when crude palm oil was first dispersed in deonized water and the extracted oil dried prior to photolysis. The dispersion was carried out in a seperatory flask, which was closed and shaken for several minutes and then allow to settle, the oil 


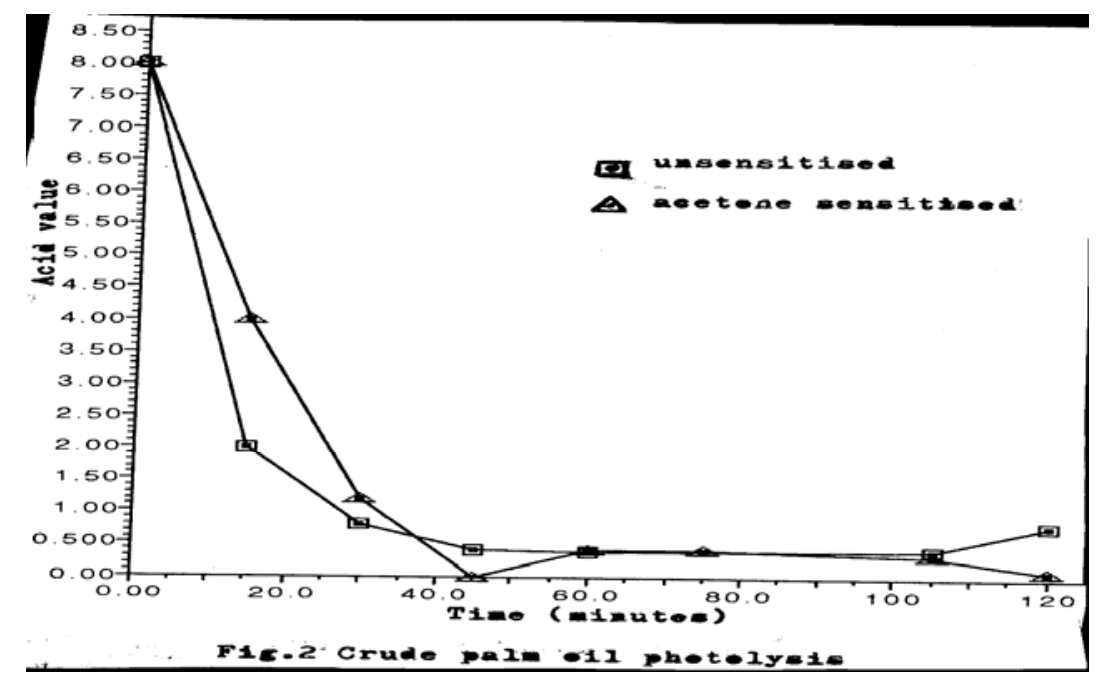

subsequently collected as the top product. Figure 4 shows similarly the result for groundnut oil in both unsensitised and acetone -sensitised photolysis.

In figure 2, the FFA (determined as AV) dropped by as much as $95 \%$ of its baseline value as a result of decarboxylation. Decarboxylation rate was estimated to be first order with respect to the FFA concentration. This trend was also maintained in the acetone sesitised system, though with a slightly decrease rate of decarboxylation. For the unsesitised system, a constant AVwas attained in the 45-105 minutes reaction time interval with a gradual drop thereafter. For the sesitised case, decarbonxylation was complete within 45 minutes. Between 45-60 minutes, the AV rose from zero by about 0.5 unit and remained constant at this level in the 60-105 minutes interval. This rise, though small, is certainly due to cleavage

of the oil to fatty acid. Concentration constancy corresponds to a situation in which acid formation and decarboxylation rates are equal. The result of figure 2 point to the complete dominance of decarboxylatoin within the first 45 minutes, while some acid formation is not necessary ruled out completely.

In figure 3, the AV of oil increased linearly and continuosely during the entire 8 hours of photolysis. Interestingly, the decarboxylation phase was either suppressed or completely eliminated in this case. Dispersion of the oil in water had the effect of removing most of its non-oil components such as sterols. Much of the three fatty acids were, however, retained as analysis prior to photolysis showed. The dominance of acid formation in this case provided us the cmpelling evidence to conclude that the non-oil component of crude palm oil play an important role in FFA decarboxylation. These 


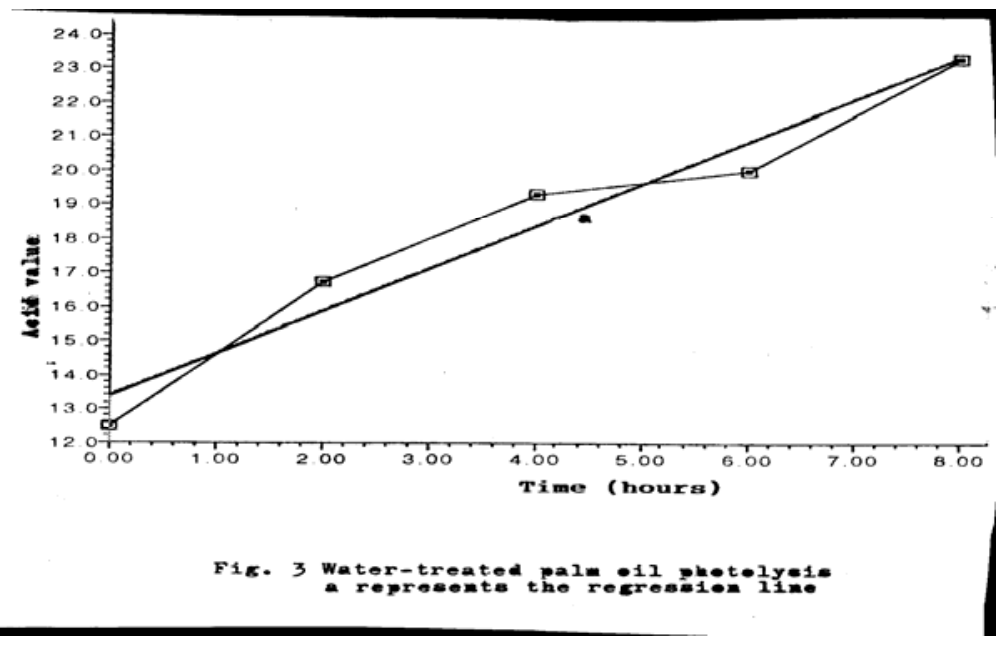

components most probably function either is raised by $36 \%$ above baseline within 10 as sensitizers or catalysts in minutes of reaction. Between 15-60 decarboxylation process.

The photochemical behavior of groundnut oil is only minimally different from that of crude palm oil. In the unsensitised photolysis (Figure 4), the AV minutes, however, the AV dropped linearly by $41 \%$ below baseline. For the sesitised system, the AV was doubled with respect to its baseline value within 5minutes of photolysis. Between 5-10 minutes, this

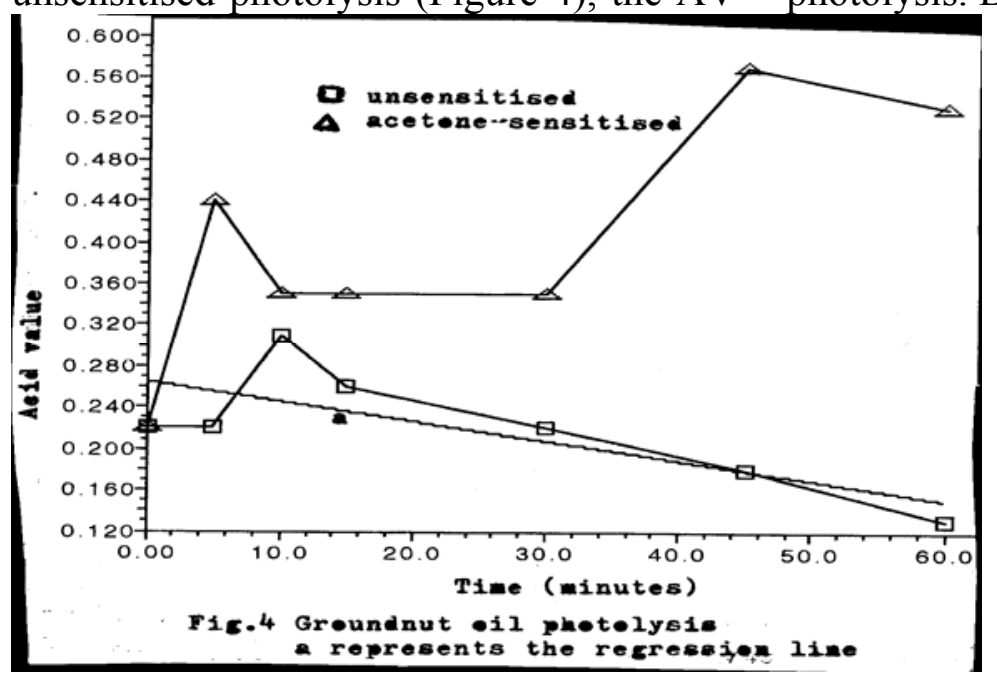

increase was only $45 \%$ above baseline. Clearly decarboxylation as a competitive reaction to acid formation is beginning to play a significant role at this point. Between 10-30 minutes, a constant AV was maintained and this respects a situation similar to that observed for crude palm oil. Rates of acid formation and decarboxylation could be assumed to be equal in time interval. In the 30-45 minutes interval, a very rapid acid formation occurred as the AV was raised by as much as $155 \%$ above baseline at this stage. In the 45-60 minutes interval, acid continued to be formed but at a somewhat diminished rate. Apparently, photosensitization enhances reaction by the obvious increase in acid formation rate over the decarboxylation rate. This observation can be understood photochemicalliy in terms of the excited state population that leads to both reactions. In the sensitized groundnut 
oil system, excited state oil molecules that leads to fatty acids are formed more rapidly than the equivalent excited state fatty acid molecules that are available for decarboxylation.

Decarboxylation occurs practically spontaneously in both unsensitised and acetone-sensitised crude palm oil photolysis. In both cases, the $\mathrm{AV}$ is reduced to near zero within 45 minutes. In the case of groundnut oil, a rise in AV was observed for both sensitized and unsensitised system within the first 5-10 minutes. Considering both oils as structurally similar, the difference in their photochemical behavior, particularly within the first 5-10 minutes, can be logically in terms of action of the non-oil components, which are present in significant concentrations in the palm oil but only in insignificant concentrations in groundnut oil. These components, including sterols, dyes and pigments, must certainly be sensitizing/catalyzing the decarboxylation reaction. Photolysis of $\mathrm{H} 2 \mathrm{O}$ - treated palm oil, from which much of the non-oil components had been removed (Figure 3), showed significant enhancement of acid formation. This result, in particular, provides a strong evidence in support of the hypothesis of the involvement of the non-oil components in the decarboxylation process.

Singlet oxygen $\left({ }^{1} 0_{2}\right)$ has been implicated severally in the photosensitized oxidation of fats, oils an unsaturated fatty acids. $5,7,8$

${ }^{1} \mathrm{O}_{2}$ is a highly reactive species, which is easily generated by photosensitization of natural pigments and dyes, for example. The following mechanistic path of its formation is often proposed.
Ch hv
$\mathrm{Ch}^{*}{ }^{3} \mathrm{O}_{2}, \mathrm{Ch}+{ }^{1} \mathrm{O}_{2}$

Here $\mathrm{Ch}$ represents the pigment or dye molecule.

For degassed reaction system (achieve by flushing with $99.9 \%$ pure $\mathrm{n} 2$ gas) no detectable decarboxylation was observed. This observation provides a strong evidence for the assumption that singlet oxygen is most probably involved in the rate- determining step of decarboxylation reaction. We have proposed the following mechanism for the reaction.

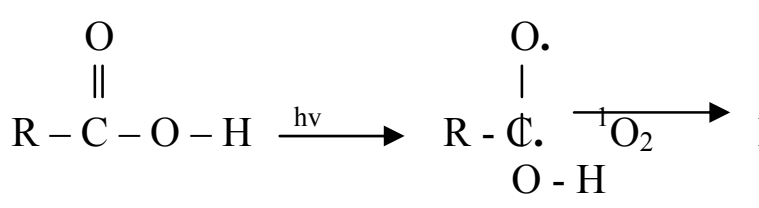

The formation of the photo chemically induced carbon-oxygen diradical is known in organic reaction system. This diradical, which is highly unstable, can react spontaneously with the highly reactive singlet oxygen to form the four-member cyclic structure. Water, which is required in trace amounts for the reaction, probably catalyses opening of the cyclic structure.

Decarboxylation, has been reported for a number of diarylacetic acids and

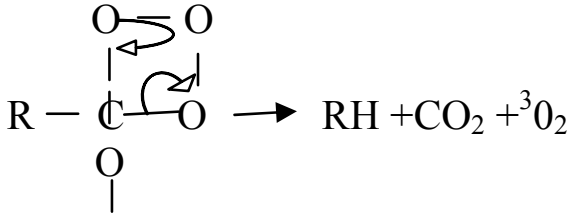

$\mathrm{H}$

pyrimidine derivatives photolysed in aqueous solutions at $\lambda=245 \mathrm{~nm} .1011$ it was observed that the excited singlet rather than the excited triplet states of the acid molecules were involved.

\section{CONCLUSION}

Decarboxylation of the free fatty acid is the major event in crude palm oil photolysis for both the unsensitisedd and 
acetone-sensitised reaction system. In the case of groundnut oil, there is a clear competition between acid formation and decarxylation reaction. Decarxylation, dominates in the unsensitised, while acid formation is the predominant reaction in acetone-sensitised system.

\section{ACKNOWLEDGEMENT}

The university of Jos senate research Grant

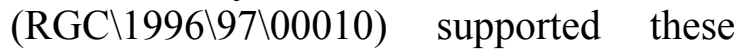
studies.

\section{REFERENCES}

1. Frankel, E. N., Neff, W.E. and Selk, E.,Lipids 16: 5 (1981) 279.

2. Gunstone, F.D., Harwood, J.L. and Padley, F.B., The lipid Handbook, $2^{\text {nd }}$ ed., p 322ff, Chapman and Hall Scientific Data Division London, 1994.

3. Labuza, T.R., CRC Critical Review, Food Technol 2 (1972) 366.
4. Cort., W.M., J. Am. Oil Chem. Soc. 51 (1974) 321.

5. Foote, C.S., Acct. Chem. Res. 1 (1968) 104.

6. Kaplan, M.L., Chem. Technol. 75 (1971) 621.

7. Rawls, H.R. and Van Santen, P.J., J. Am. Oil Chem. Soc. 47 (1970) 121.

8. Clements, A.H., Van Ben Engh, R. H., Frost, D.J., Hoogenhout, K. and Nooi, J.R., J. Am. Oil Chem. Soc. 50 (1973) 325.

9. Ekpenyong, K.I., Essien, E.E., Ibrahim, Z. and Obaje, O.J., Nig. Jour. Chem. Res. I (1996) 39.

10. Krogh, E. and wan, P., J. Am. Chem. Soc. 114 (1992) 705.

11. Wang, S.Y., Nnadi, J. C. and Greenfeld, D., Tetrahedron 26 (1970) 5913. accepted 18/9/2002

received 18/3/2002 